\title{
Bounds on double-diffusive convection
}

\author{
By NEIL J. BALMFORTH,, ${ }^{1,2}$ SHILA A. GHADGE, \\ ATICHART KETTAPUN ${ }^{3}$ AND SHREYAS D. MANDRE \\ ${ }^{1}$ Department of Mathematics, University of British Columbia, Vancouver, \\ BC, V6T 1Z2, Canada \\ ${ }^{2}$ Department of Earth and Ocean Sciences, University of British Columbia, \\ Vancouver, BC, V6T 1Z2, Canada \\ ${ }^{3}$ Department of Mathematics, University of California, Santa Cruz, CA 95064, USA
}

(Received 5 June 2005 and in revised form 3 May 2006)

We consider double-diffusive convection between two parallel plates and compute bounds on the flux of the unstably stratified species using the background method. The bound on the heat flux for Rayleigh-Bénard convection also serves as a bound on the double-diffusive problem (with the thermal Rayleigh number equal to that of the unstably stratified component). In order to incorporate a dependence of the bound on the stably stratified component, an additional constraint must be included, like that used by Joseph (Stability of Fluid Motion, 1976, Springer) to improve the energy stability analysis of this system. Our bound extends Joseph's result beyond his energy stability boundary. At large Rayleigh number, the bound is found to behave like $R_{T}^{1 / 2}$ for fixed ratio $R_{S} / R_{T}$, where $R_{T}$ and $R_{S}$ are the Rayleigh numbers of the unstably and stably stratified components, respectively.

\section{Introduction}

Mathematical models describing physical flows often have multiple possible solutions that prove difficult to find due to the complex nature of the basic equations. Worse still, such flows are often turbulent, which precludes computing some of the physically relevant solutions owing to the inability to resolve the finest scales. In this situation, it is helpful to search for other, more indirect approaches to the problem that may assist in understanding crucial characteristics of the flow. One such approach is upper bound theory, wherein one avoids the search for actual solutions, but places bounds on some of their average properties. Malkus (1954) was the first to propose this kind of idea in the context of thermal convection (the Rayleigh-Bénard problem), and Howard (1963) subsequently set the theory on a firm mathematical basis and devised techniques to calculate the bound. Whilst the governing partial differential equations (PDEs) themselves are abandoned, the method retains two integrals relations, or 'power integrals', derived from them, which is the crux of how the approach is far simpler than direct computations.

Making use of clever inequalities, Howard deduced rigorous but rough bounds on the heat flux that scaled like $R_{T}^{1 / 2}$, where $R_{T}$ is the Rayleigh number. Howard also computed bounds using test functions with a single horizontal wavenumber, which leads to the alternative scaling, $R_{T}^{3 / 8}$, for large $R_{T}$. The single-wavenumber bound is only valid if other functions do not lead to a higher value of the heat flux, which Busse (1969) later showed to be the case at sufficiently high Rayleigh number. He also generalized Howard's approach using an elaborate procedure to account for more than one wavenumber, and recovered the $R_{T}^{1 / 2}$ scaling in the infinite Rayleigh number limit. 
Howard's method centres on a decomposition of all the physical fields into horizontal averages and fluctuations about them. In an alternative approach, the Constantin-Doering-Hopf (CDH) background method (Doering \& Constantin 1996), the variables are also decomposed, but this time exploiting arbitrary 'background' fields. Integral identities similar to the ones used in Howard's method are constructed, and the choice for the background is dictated by constraints similar to those obtained in energy stability theory (see Joseph 1976). Work by Nicodemus, Grossmann \& Holthaus (1997) and Kerswell (1998) has proved that, although the Howard-Busse and the $\mathrm{CDH}$ methods appear different, they are complementary or optimal duals of each other, and ultimately lead to the same result.

In this article, we use the background method to place bounds on double-diffusive convection (i.e. convection resulting from the dependence of buoyancy on two properties that diffuse at different rates). Such systems are often termed thermohaline, referring to their most common occurrence in oceans and other large water bodies, with salt and heat playing the roles of the two components. Interesting dynamics ensues when the two components affect the density stratification in opposite senses, and convection may occur even when the total density gradient is gravitationally stable (Veronis 1965; Baines \& Gill 1969). For example, near the polar ice caps, melting of ice releases fresh but cold water near the surface, a situation prone to oscillatory double-diffusive (ODD) convection (Jacobs et al. 1981; Neal, Neshira \& Denner 1969). ODD convection is also thought to occur in meddies (vortices of warm, salty water commonly observed in the East Atlantic emanating from the Mediterranean, Ruddick (1992)). In an astrophysical context, ODD convection is believed to operate in the interiors of older stars where the two components are entropy and the elements produced by thermonuclear reactions (Spiegel 1969); mixing by ODD convection may replenish the reactive core of the star with fresh fuel and thus affect its evolution.

The opposite case, in which salt stratification is destabilizing but heat is stabilizing, is susceptible to the formation of salt fingers. Stern (1969) proposed that enhanced fluxes resulting from these fingers are instrumental in forming the staircase-like salinity profiles observed in laboratory experiments and the open ocean. The articles by Merryfield (2000) and Schmitt (1994) provide recent reviews on this subject. Analogues of salt fingers have also been suggested to arise in some astrophysical situations, where the role of salt is played by locally overabundant heavier elements such as helium (Ulrich 1972; Vauclair 2004). In all these applications quantifying the degree of mixing generated by thermohaline processes is paramount, which highlights the importance of characterizing the flux laws in double-diffusive convection, especially in the turbulent regime. Whilst the desired characterization of the flux laws remains elusive to analysis, at least at present, we follow Malkus's vision and calculate an upper bound on the flux of the unstably stratified species.

For double diffusion, this bounding exercise has two key novelties compared to the Rayleigh-Bénard problem. First, at the onset of convection, double-diffusive systems show a richer array of dynamics than purely thermal systems. In RayleighBénard convection, when the system first becomes convectively unstable, a branch of steady convection solutions bifurcates supercritically from the motionless state; that is, there is a smooth onset to steady overturning. This simple scenario does not carry over to the double-diffusive case: as one raises $R_{T}$ to drive the system into convection, the linear instability can take the form of either steady overturning or oscillatory convection. Furthermore, the steady bifurcation can become subcritical, which implies the existence of multiple finite-amplitude solutions at lower Rayleigh number that must, in turn, appear in saddle-node bifurcations at yet lower $R_{T}$. 
The existence of multiple solutions demands that the conductive state be subject to finite-amplitude instability, even if it is linearly stable. All such dynamics must become embedded in the upper bound, which may even jump discontinuously at the saddle-node bifurcations. This raises the interesting question of whether the upper bound theory can be used to detect and characterize finite-amplitude instability and saddle-node bifurcations.

Second, the bounding exercise also has some interesting mathematical twists. We first show that the upper bound obtained on the flux of the unstably stratified component in the absence of the other component also serves as a bound in the presence of that stabilizing field. In fact, this is the result that appears when we extend the background method in a straightforward way to doubly-diffusive convection. Whilst this result is heuristically expected, since the stratification of the stable component can only diminish the flux, it fails to provide a dependence on both components. In previous attempts, Lindberg (1971) and Straus (1973) used variants of the singlewavenumber approach to bound the ODD and salt-fingering cases, respectively. In order to obtain a non-trivial dependence of the bound on the salt flux, Lindberg maximized the heat and salt fluxes simultaneously. Not only is there no reason to expect a single wavenumber, there is also no justification for assuming the fluxes to be maximal simultaneously. The procedure yielded bounds that scaled like $R_{T}^{3 / 8}$, where $R_{T}$ now denotes the Rayleigh number of the unstably stratified component. Straus exploited the large difference between the diffusivities of heat and salt to solve the heat equation asymptotically, thereby building the full effects of the stabilizing component into the bounding formulation automatically. But like Lindberg's, Straus's bound also scales with the $3 / 8$ power of $R_{T}$, and again reflects the inadequacy of a single wavenumber. In the current work, we identify one more integral constraint on double-diffusive convection, which Joseph (1976) has shown to be crucial in energy stability analysis. By augmenting the upper bound analysis with this integral constraint, and avoiding the use of a single wavenumber, we capture the effect of both components and construct a true bound. A similar analysis was also presented by Stern (1982).

\section{Mathematical formulation}

As is traditionally done, we model our system by the Boussinesq equations:

$$
\begin{aligned}
\boldsymbol{u}_{t}+\boldsymbol{u} \cdot \nabla \boldsymbol{u} & =-\frac{\nabla p}{\rho}+g\left(\alpha_{T} T-\alpha_{S} S\right) \hat{z}+\nu \nabla^{2} \boldsymbol{u}, \\
T_{t}+\boldsymbol{u} \cdot \nabla T & =\kappa_{T} \nabla^{2} T, \\
S_{t}+\boldsymbol{u} \cdot \nabla S & =\kappa_{S} \nabla^{2} S, \\
\nabla \cdot \boldsymbol{u} & =0
\end{aligned}
$$

where $\boldsymbol{u}(\boldsymbol{x}, t)$ is an incompressible velocity field, and $T$ and $S$ represent two scalar components that affect the density of the fluid. We only deal with the situation in which the two components affect the density stratification in opposite senses; without loss of generality, we set $T$ to be unstably stratified and $S$ to be stably stratified. If the diffusivity of $T\left(\kappa_{T}\right)$ is larger than that of $S\left(\kappa_{S}\right)$ the system is susceptible to ODD convection. In the opposite case, the system is susceptible to ' $T$-fingers' (because in this case $T$ is playing the role of salt). The other physical parameters are the acceleration due to gravity $(g)$, coefficients of expansion due to variations in $S\left(\alpha_{S}\right)$ and $T\left(\alpha_{T}\right)$, and the kinematic viscosity $(v)$. 
We prescribe the values of $S$ and $T$ at the two boundaries, $z=0$ and $z=H$ :

$$
\begin{array}{ll}
T(z=H)=T_{\text {top }}, & T(z=0)=T_{\text {top }}+\Delta T, \\
S(z=H)=S_{\text {top }}, & S(z=0)=S_{\text {top }}+\Delta S .
\end{array}
$$

For the velocity field, we either use the no-slip condition,

$$
\boldsymbol{u}=\mathbf{0},
$$

or stress-free conditions,

$$
w=0, \quad u_{z}=v_{z}=0 .
$$

Both these cases are considered when calculating the bound. However, the linear stability and some nonlinear solutions that we present are computed using the stressfree conditions, mostly for computational convenience.

We place the equations in dimensionless form by rescaling,

$$
\left.\begin{array}{ll}
u \rightarrow \frac{\kappa_{T}}{H} \boldsymbol{u}, & T-T_{\text {top }} \rightarrow \Delta T T, \quad S-S_{\text {top }} \rightarrow \Delta S S, \quad \boldsymbol{x} \rightarrow H \boldsymbol{x}, \\
t \rightarrow \frac{H^{2}}{\kappa_{T}} t, & p-\rho g\left(\alpha_{T} T_{\text {top }}-\alpha_{S} S_{\text {top }}\right) z \rightarrow \frac{\rho \kappa_{T}^{2}}{H^{2}} p .
\end{array}\right\}
$$

This gives rise to four dimensionless numbers,

$$
R_{T}=\frac{g \alpha_{T} \Delta T H^{3}}{\nu \kappa_{T}}, \quad R_{S}=\frac{g \alpha_{S} \Delta S H^{3}}{\nu \kappa_{S}}, \quad \operatorname{Pr}=\frac{\nu}{\kappa_{T}}, \quad \beta=\frac{\kappa_{S}}{\kappa_{T}},
$$

and the governing equations become

$$
\begin{aligned}
\frac{1}{P r}\left(\boldsymbol{u}_{t}+\boldsymbol{u} \cdot \nabla \boldsymbol{u}+\nabla p\right) & =\left(R_{T} T-\beta R_{S} S\right) \hat{z}+\nabla^{2} \boldsymbol{u}, \\
T_{t}+\boldsymbol{u} \cdot \nabla T & =\nabla^{2} T, \\
S_{t}+\boldsymbol{u} \cdot \nabla S & =\beta \nabla^{2} S, \\
\nabla \cdot \boldsymbol{u} & =0 .
\end{aligned}
$$

The accompanying boundary conditions are

$$
\left.\begin{array}{l}
T(z=1)=S(z=1)=0, \\
T(z=0)=S(z=0)=1,
\end{array}\right\}
$$

plus (2.7) or (2.8) on $z=0$ and $z=1$ and periodicity in $x$ and $y$.

\section{Energy stability}

We start with the criteria for nonlinear stability of the purely conductive state of this system. A very brief account of this analysis was given by Joseph (1976). We elaborate and build upon Joseph's results here in order to offer a more complete discussion and extract some important physical results; in doing so, we also emphasize the key connection with the bounding theory to follow.

\subsection{Mathematical details}

Consider $\boldsymbol{u}=\mathbf{0}+\boldsymbol{u}(\boldsymbol{x}, t), \quad T=T_{0}+\theta(\boldsymbol{x}, t), \quad S=S_{0}+\sigma(\boldsymbol{x}, t)$, and $p=P_{0}+\Pi$, where $T_{0}=S_{0}=1-z$ and $P_{0}=\operatorname{Pr}\left(R_{T}-\beta R_{S}\right)\left(z-z^{2} / 2\right)$ characterize the purely conductive solution of (2.11)-(2.14), and $\boldsymbol{u}, \theta, \sigma$ and $\Pi$ are arbitrary perturbations. The 
perturbations satisfy

$$
\begin{aligned}
\frac{1}{P r}\left(\boldsymbol{u}_{t}+\boldsymbol{u} \cdot \nabla \boldsymbol{u}+\nabla \Pi\right) & =\left(R_{T} \theta-\beta R_{S} \sigma\right) \hat{z}+\nabla^{2} \boldsymbol{u}, \\
\theta_{t}+\boldsymbol{u} \cdot \nabla \theta-w & =\nabla^{2} \theta, \\
\sigma_{t}+\boldsymbol{u} \cdot \nabla \sigma-w & =\beta \nabla^{2} \sigma, \\
\nabla \cdot \boldsymbol{u} & =0 .
\end{aligned}
$$

The kinetic energy equation is constructed by taking the dot product of the momentum equation (3.1) with $\boldsymbol{u}$ and integrating over the domain,

$$
\frac{1}{2 P r}\left\langle|\boldsymbol{u}|^{2}\right\rangle_{t}=-\left\langle|\nabla \boldsymbol{u}|^{2}\right\rangle+R_{T}\langle\theta w\rangle-\beta R_{S}\langle\sigma w\rangle,
$$

where

$$
\langle\cdots\rangle \equiv \frac{1}{4 L_{x} L_{y}} \int_{-L_{y}}^{L_{y}} \int_{-L_{x}}^{L_{x}} \int_{0}^{1} \cdots \mathrm{d} z \mathrm{~d} x \mathrm{~d} y,
$$

$2 L_{x}$ and $2 L_{y}$ are the periodicities in $x$ and $y$, respectively, and $|\nabla \boldsymbol{u}|^{2}=\nabla \boldsymbol{u}: \nabla \boldsymbol{u}^{T}$. Similarly, by multiplying (3.2) and (3.3) by $\theta$ and $\sigma$, respectively, and integrating, we arrive at the following power integrals:

$$
\begin{aligned}
\frac{1}{2}\left\langle\theta^{2}\right\rangle_{t} & =-\left\langle|\nabla \theta|^{2}\right\rangle+\langle\theta w\rangle, \\
\frac{1}{2}\left\langle\sigma^{2}\right\rangle_{t} & =-\beta\left\langle|\nabla \sigma|^{2}\right\rangle+\langle\sigma w\rangle .
\end{aligned}
$$

While these integral equations are the obvious generalization of those used for the energy stability for thermal convection, there is a less obvious integral which is also crucial. It is constructed by multiplying (3.2) by $\sigma$ and adding it to the product of (3.3) and $\theta$ and integrating:

$$
\langle\theta \sigma\rangle_{t}=\langle(\theta+\sigma) w\rangle-(1+\beta)\langle\nabla \theta \cdot \nabla \sigma\rangle .
$$

The optimal way in which to combine these integral equations so as to yield the best stability criterion is the essence of the analysis. Since we do not know the optimal combination a priori, we start with an arbitrary linear combination of (3.5)-(3.8) and arrive at the generalized energy equation:

$$
\begin{aligned}
E_{t}=-\left\langle|\nabla \boldsymbol{u}|^{2}\right\rangle-\lambda_{T}^{2} & R_{T}\left\langle|\nabla \theta|^{2}\right\rangle-\beta \lambda_{S}^{2} R_{S}\left\langle|\nabla \sigma|^{2}\right\rangle+\lambda_{T} R_{T} b_{T}\langle\theta w\rangle \\
& +\frac{\sqrt{\beta} \lambda_{S} R_{S} b_{S}}{\alpha}\langle\sigma w\rangle-(1+\beta) c \lambda_{T} \lambda_{S} \sqrt{R_{T} R_{S}}\langle\nabla \theta \cdot \nabla \sigma\rangle,
\end{aligned}
$$

where

$$
\begin{gathered}
\alpha^{2}=R_{S} / R_{T}, \\
E \equiv \frac{1}{2 P r}\left\langle|\boldsymbol{u}|^{2}\right\rangle+\frac{\lambda_{T}^{2} R_{T}}{2}\left\langle\theta^{2}\right\rangle+\frac{\lambda_{S}^{2} R_{S}}{2}\left\langle\sigma^{2}\right\rangle+c \lambda_{T} \lambda_{S} \sqrt{R_{T} R_{S}}\langle\theta \sigma\rangle, \\
b_{T} \equiv \frac{1}{\lambda_{T}}+\lambda_{T}+c \alpha \lambda_{S}, \\
b_{S} \equiv-\frac{\sqrt{\beta} \alpha}{\lambda_{S}}+\frac{\alpha}{\sqrt{\beta}} \lambda_{S}+\frac{c}{\sqrt{\beta}} \lambda_{T},
\end{gathered}
$$

and $\lambda_{T}, \lambda_{S}$ and $c$ are the constants used to form the combination. When $R_{S}=0$ the effect of $S$ disappears and we recover the result for thermal convection. The basic state is said to be 'energy stable' when the energy-norm, $E$, of the perturbations is 
positive definite and decays monotonically (the right-hand side of (3.9) is negative definite) for all possible perturbations. It is straightforward to show that $E$ in (3.11) is positive definite when $|c|<1$.

First, we demonstrate the inability of (3.5)-(3.7) to capture the stabilizing effect of $R_{S}$ when $c=0$. In this case, the energy equation takes the form,

$$
\begin{aligned}
E_{t}= & R_{T}\left(1+\lambda_{T}^{2}\right)\langle\theta w\rangle+\alpha^{2} R_{T}\left(-\beta+\lambda_{S}^{2}\right)\langle\sigma w\rangle \\
& -R_{T}\left(\lambda_{T}^{2}\left\langle|\nabla \theta|^{2}\right\rangle+\alpha^{2} \beta \lambda_{S}^{2}\left\langle|\nabla \sigma|^{2}\right\rangle\right)-\left\langle|\nabla \boldsymbol{u}|^{2}\right\rangle
\end{aligned}
$$

and we refer to $E$ as the 'regular' energy. The optimization problem of finding the critical $R_{T}$ leads to the criterion for stability,

$$
R_{T}<R_{T c}=\frac{4 R_{c}}{F\left(\lambda_{T}, \lambda_{S}\right)},
$$

where $R_{c}$ is the critical Rayleigh number for the onset of thermal convection and

$$
F\left(\lambda_{T}, \lambda_{S}\right)=\left(\frac{1}{\lambda_{T}}+\lambda_{T}\right)^{2}+\left(-\frac{\beta}{\lambda_{S}}+\lambda_{S}\right)^{2} .
$$

The choice of boundary conditions on the velocity enters the consideration through the value of $R_{c}$. For no slip, $R_{c} \approx 1707$, whereas for the stress-free condition $R_{c} \approx 657$. We now choose $\lambda_{T}$ and $\lambda_{S}$ to maximize the range of $R_{T}$ for which perturbations decay. That is, we look for the minimum value of the function $F\left(\lambda_{T}, \lambda_{S}\right)$, which occurs for $\lambda_{S}^{2}=\beta$ and $\lambda_{T}=1$, giving $R_{T c}=R_{c}$, as stated earlier. To improve this stability condition we must take $c \neq 0$, thereby including (3.8).

Going back to (3.9), the terms involving $\nabla \theta$ and $\nabla \sigma$ are negative semi-definite only if $c \leqslant 2 \sqrt{\beta} /(1+\beta)$. We choose $c=2 \sqrt{\beta} /(1+\beta)$ and then combine all three terms into $\left\langle|\nabla f|^{2}\right\rangle$, where

$$
f \equiv \lambda_{T} \sqrt{R_{T}} \theta+\lambda_{S} \sqrt{\beta R_{S}} \sigma .
$$

This leaves us with just two sign-indefinite terms, $\langle\theta w\rangle$ and $\langle\sigma w\rangle$. This pair can only be bounded if they can again be grouped together in the combination $f$, which prompts the constraint

$$
b_{T}=b_{S}
$$

The energy equation now takes the form

$$
E_{t}=R_{T}^{1 / 2} b_{T}\langle f w\rangle-\left\langle|\nabla f|^{2}\right\rangle-\left\langle|\nabla \boldsymbol{u}|^{2}\right\rangle,
$$

which is very similar to the one obtained for thermal convection, but with a modified thermal Rayleigh number and the field $f$ playing the role of temperature. Again finding the optimal perturbation, we conclude that the condition for energy stability is

$$
R_{T}<R_{T c}=\frac{4 R_{c}}{b_{T}^{2}} .
$$

We still have the freedom to choose one of either $\lambda_{T}$ or $\lambda_{S}$ so as to obtain the best possible stability criterion. This leads to the following minimization problem for the critical thermal Rayleigh number $\left(R_{T c}\right)$ :

$$
R_{T c}=4 R_{c}\left(\min _{b_{T}=b_{S}} b_{T}\left(\lambda_{T}, \lambda_{S}\right)^{2}\right)^{-1} .
$$


The details of the minimization are given in the Appendix. The resulting stability condition can be encapsulated in the formulae

$$
R_{T c}= \begin{cases}R_{c}+R_{S} & \text { if } \alpha \leqslant \beta<1 \text { or } \beta \geqslant 1>\alpha \\ \left(\sqrt{R_{c}\left(1-\beta^{2}\right)}+\beta \sqrt{R_{S}}\right)^{2} & \text { if } \beta<\alpha<\beta^{-1} \\ \infty & \text { if } \alpha \geqslant \beta^{-1}>1 \text { or } \alpha \geqslant 1 \text { and } \beta \geqslant 1,\end{cases}
$$

which were derived previously by Joseph.

\subsection{Interpretation for stress-free plates}

Now we draw some conclusions from Joseph's result and compare with linear stability theory, specifically for the case of stress-free plates. The linear stability theory is described by Veronis (1965) and can be summarized as follows: linear instability can appear as either steady or oscillatory convection, the corresponding critical Rayleigh numbers being given by

$$
\begin{aligned}
\text { steady: } & R_{T}=R_{S}+R_{c}, \quad \text { if } \alpha^{2} \frac{\beta+P r}{1+P r}<\beta<1 \text { or } \beta>1 \\
\text { oscillatory: } & R_{T}=(P r+\beta)\left(\frac{\beta R_{S}}{1+P r}+\frac{R_{c}(1+\beta)}{P r}\right) \quad \text { if } \alpha^{2} \frac{\beta+P r}{1+P r}>\beta \text { and } \beta<1 .
\end{aligned}
$$

In figure 1, the energy stability condition (3.21) is compared with the conditions for the onset of linear and nonlinear instability. First consider the fingering case $(\beta>1)$, represented in figure $1(a)$, which is the same for all values of $\beta$ and $\operatorname{Pr}$. In this case, the conductive state becomes linearly unstable to steady convection on the line (3.22), and is never unstable to oscillatory convection. The energy stability threshold agrees with linear onset everywhere, proving that all perturbations, irrespective of their size, should decay below that line.

The ODD case $(\beta<1)$ is rather more complicated, and the dynamics of the system depends on the detailed parameter settings. Figures $1(b)$ and $1(c)$ show a representative case with $\beta=0.5$ and $P r=2$; once we fix those parameters, the behaviour of the system is determined by where it falls on the $\left(R_{S} / R_{c}, R_{T} / R_{c}\right)$-plane, and the range of possibilities is delimited by the four curves shown in the figure. For $\alpha<\sqrt{\beta(1+P r) /(\beta+P r)}$ (left of point B), steady convection appears on the line (3.22) and is the only linear instability. For $\alpha<\beta$, or to the left of point A, the energy stability condition in (3.21) also agrees with steady onset. However, to the right of that point, the two conditions diverge from one another, indicating that energy stability is lost before the motionless state becomes linearly unstable to steady convection. The steady instability is further superceded by the onset of oscillatory convection for $\alpha>\sqrt{\beta(1+P r) /(\beta+P r)}$, or to the right of point B in figure $1(c)$. Moreover, except at one special point (labelled D) where the two curves are tangential, energy stability never agrees with linear oscillatory instability. In other words, only over a limited parameter range does the loss of energy stability correspond to the onset of linear instability, in contrast to thermal convection and the fingering case, where they always agree.

Part of the reason for the disagreement between the energy stability condition and linear onset arises because the steady bifurcation becomes subcritical at point A. To the right of this point, the subcritical instability leads to steady convection solutions even in the linearly stable regime. These steady convection solutions do not persist very far below the steady linear stability line because they turn around at a saddle-node bifurcation (Veronis 1965). The saddle node in the past has been located 

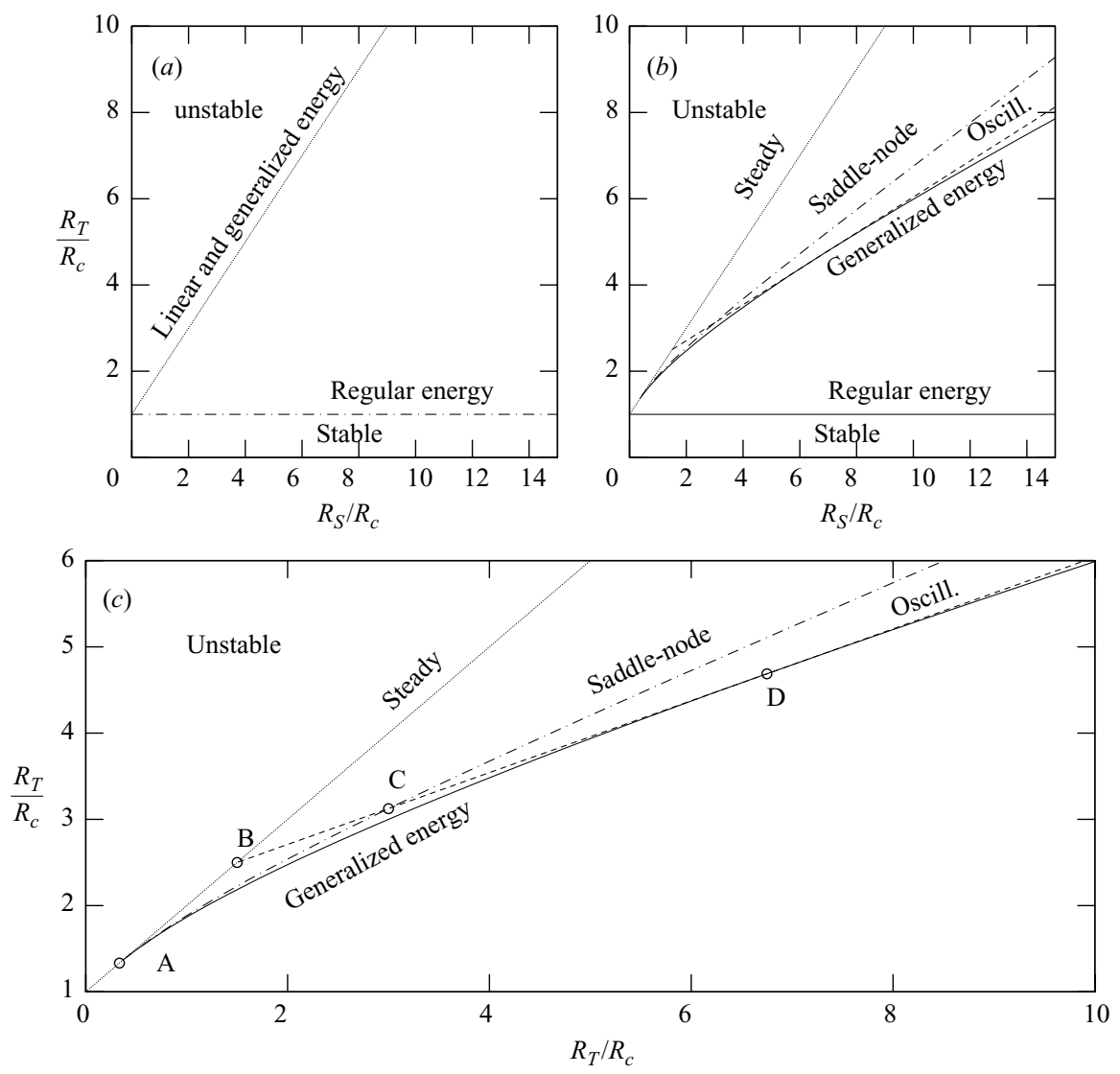

Figure 1. Stability boundaries on the $\left(R_{S} / R_{c}-R_{T} / R_{c}\right)$-plane. $(a)$ The fingering case $(\beta>1)$, where the only linear instability is that of steady convection and the generalized energy stability condition agrees with it (topmost curve). The curve below it shows the regular energy stability criterion. These curves do not depend on the precise values of $\beta$ and $P r$. (b) The ODD case $(\beta=0.5$ and $\operatorname{Pr}=2)$ and to clarify the details a magnified view is plotted in $(c)$. The topmost solid line corresponds to the onset of steady convection which is supercritical to the left of point A and subcritical to its right. The unstable branch bifurcating from the subcritical bifurcation turns around at a saddle-node bifurcation whose location is shown by the dashed-dotted line. The nonlinear solutions at the saddle-node are calculated by expanding the variables in a truncated Fourier series in $x$ and a sine series in $z$. The dashed line shows the linear stability criterion for onset of oscillatory convection. The solid lines again show the generalized and regular energy stability conditions, respectively.

using a crude Galerkin truncation of the governing equations. To improve upon this, we have accurately computed the locus of that bifurcation numerically via Fourier expansion and a continuation algorithm. The locus is plotted in figure 1. In the region between this locus and the onset of steady convection, multiple steady solutions are guaranteed.

When multiple solutions exist, certain finite-amplitude perturbations and the energy associated with them will not decay to zero but saturate to a finite value, reflecting a transition to one of the other solutions. As a result, the energy stability condition cannot agree with the onset of linear instability whenever there are multiple solutions. Indeed, we find that the energy stability condition is tangential to the saddle-node line at point A, suggesting that the saddle-node is the cause of the loss of energy 
stability there. However, as $R_{S}$ increases, the saddle-node line and energy stability condition diverge, indicating some other reason for the loss of energy stability. The saddle-node line also crosses the threshold for the onset of oscillatory convection (point $\mathrm{C}$ in figure 1), whereupon oscillatory nonlinear solutions come into existence before the saddle-node. Unlike steady convection, the onset of oscillatory convection is always supercritical (Veronis 1965). Nevertheless, the energy stability condition disagrees with oscillatory onset except at one point. This leaves us with a significant discrepancy between the energy stability condition and either the saddle-node line or the oscillatory onset.

The discrepancy could arise from three possible sources, amongst which we are currently unable to distinguish. First, there could be other unidentified nonlinear solutions lying below the computed saddle-node line. The detection of such additional multiple equilibria would require an intensive search of the solution space of the governing equations at each point on the parameter plane. However, our original purpose was to avoid such a time-consuming open-ended exercise, and hence we will not pursue this.

The second possibility is that the power integrals included in the energy stability formulation allow a wider class of trial functions than are solutions to the governing equations. Above the energy stability condition, the energy method indicates that there are trial functions for which the generalized energy grows in time, yet these may not be real solutions. The cure is to better constraint the function space by, for example, adding more power integrals. A curious observation arises on exploring in more detail the point of intersection of the energy stability condition and the linear oscillatory onset. The former is independent of Prandtl number, but the latter is not. Yet, when one constructs the envelope of the oscillatory onset line for all possible Prandtl numbers, the energy stability condition is recovered exactly. This suspicious coincidence leaves one wondering whether the main problem is the lack of Prandtlnumber dependence in the energy stability condition, which could be alleviated by building in extra constraints.

The final possibility is transient amplification. This is a purely linear mechanism wherein the energy norm chosen to determine stability grows initially for certain initial conditions. The growth can be attributed to the presence of non-orthogonal linear modes, even when each of these modes decays exponentially (Baggett, Driscoll $\&$ Trefethen 1993; Waleffe 1995). In the thermohaline context, transient amplification has been invoked in studies of ocean circulation (Tziperman \& Ioannou 2002; Dijkstra, Te Raa \& Weijer 2004), and with regard to possible transitions in the paleoclimate (Bryan 1986; Stocker 1999). As far as energy stability is concerned, a sub-optimal choice of the energy norm may lead to transient growth even in situations for which there is no finite-amplitude instability. Indeed, this is exactly what happens with the regular energy norm for $R_{T c}>R_{T}>R_{c}$. The remedy is to generalize the energy norm and curb transient amplification, leading to the improved energy stability condition. But even this generalized energy stability condition may not correspond to a finite-amplitude instability as all perturbations may still eventually decay beyond this condition. Whether a true finite-amplitude instability criterion can be derived from power-integral considerations remains an open question.

\section{The background method}

Energy stability rigorously predicts there to be no convective motion when $R_{T}<R_{T c}$, but the analysis provides no predictions for larger $R_{T}$. More information can be gained 
by employing the background method to find a bound on a flow property like the average species transport over long times. We undertake this calculation in this section.

\subsection{The general formulation}

The average transport of $T$ is quantified by the Nusselt number $(N u)$, defined as

$$
N u=\lim _{t \rightarrow \infty} \frac{1}{4 L_{x} L_{y} t} \int_{0}^{t} \int_{-L_{y}}^{L_{y}} \int_{-L_{x}}^{L_{x}} T_{z}(z=1) \mathrm{d} y \mathrm{~d} x \mathrm{~d} t .
$$

A volume integration of (2.12) multiplied by $T$ puts the Nusselt number in a more usable form:

$$
N u=\left\langle|\nabla T|^{2}\right\rangle
$$

where we now redefine $\langle\cdots\rangle$ to include a long time average:

$$
\langle\cdots\rangle \equiv \lim _{t \rightarrow \infty} \frac{1}{4 L_{x} L_{y} t} \int_{0}^{t} \int_{-L_{y}}^{L_{y}} \int_{-L_{x}}^{L_{x}} \int_{0}^{1} \cdots \mathrm{d} z \mathrm{~d} x \mathrm{~d} y \mathrm{~d} t .
$$

The $T$ and $S$ fields are decomposed into backgrounds and fluctuations as

$$
T(\boldsymbol{x}, t)=1-z+\phi(z)+\theta(\boldsymbol{x}, t), \quad S(\boldsymbol{x}, t)=1-z+\psi(z)+\sigma(\boldsymbol{x}, t),
$$

where we denote the backgrounds by $\phi$ and $\psi$ and the fluctuations by $\theta$ and $\sigma$. With this selection, $\phi(z), \theta(\boldsymbol{x}, t), \psi(z)$ and $\sigma(\boldsymbol{x}, t)$ satisfy homogeneous boundary conditions. The decomposition is arbitrary at the moment but will be made unique as the analysis proceeds. With the decomposition, we construct the power integrals:

$$
\begin{aligned}
R_{T}\langle\theta w\rangle-\beta R_{S}\langle\sigma w\rangle-\left\langle|\nabla \boldsymbol{u}|^{2}\right\rangle & =0, \\
\left\langle\left(1-\phi^{\prime}\right) \theta w\right\rangle-\left\langle\phi^{\prime} \theta_{z}\right\rangle-\left\langle|\nabla \theta|^{2}\right\rangle & =0, \\
\left\langle\left(1-\psi^{\prime}\right) \sigma w\right\rangle-\beta\left\langle\psi^{\prime} \sigma_{z}\right\rangle-\beta\left\langle|\nabla \sigma|^{2}\right\rangle & =0, \\
\left\langle\left(1-\phi^{\prime}\right) \sigma w+\left(1-\psi^{\prime}\right) \theta w\right\rangle-\left\langle\phi^{\prime} \sigma_{z}\right\rangle-\beta\left\langle\psi^{\prime} \theta_{z}\right\rangle-(1+\beta)\langle\nabla \sigma \cdot \nabla \theta\rangle & =0,
\end{aligned}
$$

where primes denote differentiation with respect to $z$. To find a bound, we relax the condition that $\boldsymbol{u}, \theta$ and $\sigma$ solve the governing PDEs, but require them to satisfy the above integral relations. As will be shown later, the inclusion of the equation (4.8) is crucial in obtaining the dependence of the bound on $R_{S}$ in the same way it was needed for energy stability.

The method proceeds by writing a variational problem in which we maximize the Nusselt number subject to the integral constraints. Thus, we consider the Lagrangian,

$$
\begin{aligned}
\mathscr{L}[\boldsymbol{u}, \theta, \sigma]= & +\left\langle\phi^{\prime 2}\right\rangle+2\left\langle\phi^{\prime} \theta_{z}\right\rangle+\left\langle|\nabla \theta|^{2}\right\rangle+\sqrt{a}\langle\Pi(\boldsymbol{x}) \nabla \cdot \boldsymbol{u}\rangle \\
& +a\left[\left(R_{T}\langle\theta w\rangle-\beta R_{S}\langle\sigma w\rangle-\left\langle|\nabla \boldsymbol{u}|^{2}\right\rangle\right]\right. \\
& +a \lambda_{T}^{2} R_{T}\left[\left\langle\left(1-\phi^{\prime}\right) \theta w\right\rangle-\left\langle\phi^{\prime} \theta_{z}\right\rangle-\left\langle|\nabla \theta|^{2}\right\rangle\right] \\
& +a \lambda_{S}^{2} R_{S}\left[\left\langle\left(1-\psi^{\prime}\right) \sigma w\right\rangle-\beta\left\langle\psi^{\prime} \sigma_{z}\right\rangle-\beta\left\langle|\nabla \sigma|^{2}\right\rangle\right] \\
& +a c \lambda_{T} \lambda_{S} \alpha R_{T}\left\langle\left(1-\phi^{\prime}\right) \sigma w+\left(1-\psi^{\prime}\right) \theta w-\phi^{\prime} \sigma_{z}-\beta \psi^{\prime} \theta_{z}\right. \\
& -(1+\beta) \nabla \sigma \cdot \nabla \theta\rangle,
\end{aligned}
$$

where $a, \lambda_{T}, \lambda_{S}$ and $c$ are constant Lagrange multipliers, and $\Pi$ is a spatially dependent multiplier that enforces fluid incompressibility. One can verify that if $c$ is chosen to be zero, thus avoiding the constraint (4.8), the best value for $\lambda_{S}$ turns out to be $\sqrt{\beta}$ and the problem reduces to that of thermal convection. That is, the effect of $R_{S}$ disappears from the bound as in energy stability theory. We therefore retain $c$, but resist making the same choice for $c$ as in energy stability theory. Instead, we 
substitute $c=2 q \sqrt{\beta} \sqrt{1-\epsilon^{2}} /(1+\beta)$, where $q$ is a parameter $(q=1$ corresponds to the choice of energy stability theory). For algebraic convenience, we further rescale the backgrounds and fluctuations as

$$
\boldsymbol{u} \rightarrow \frac{1}{\sqrt{a}} \boldsymbol{u}, \quad \theta \rightarrow \epsilon \theta, \quad \phi \rightarrow \epsilon \phi, \quad \sigma \rightarrow \eta \sigma, \quad \psi \rightarrow \eta \psi
$$

where $\epsilon \equiv 1 /\left(\lambda_{T} \sqrt{a R_{T}}\right)$ and $\eta \equiv 1 /\left(\lambda_{S} \sqrt{a \beta R_{S}}\right)$. Then $\mathscr{L}[\boldsymbol{u}, \theta, \sigma]$ can be written as

$$
\begin{aligned}
\mathscr{L}[\boldsymbol{u}, \boldsymbol{\Theta}]= & 1+\epsilon^{2}\left\langle\phi^{\prime 2}\right\rangle-\left\langle|\nabla \boldsymbol{u}|^{2}\right\rangle-\left\langle\frac{\partial \boldsymbol{\Theta}^{T}}{\partial z} \boldsymbol{P} \boldsymbol{\Psi}^{\prime}\right\rangle \\
& +R_{T}^{1 / 2}\left\langle\left(B_{T} \theta+B_{S} \sigma\right) w\right\rangle-\left\langle\frac{\partial \boldsymbol{\Theta}^{T}}{\partial x_{i}} \boldsymbol{R} \frac{\partial \boldsymbol{\Theta}}{\partial x_{i}}\right\rangle+\langle\Pi \nabla \cdot \boldsymbol{u}\rangle,
\end{aligned}
$$

where

$$
\begin{aligned}
& \boldsymbol{\Theta} \equiv\left(\begin{array}{l}
\theta \\
\sigma
\end{array}\right), \quad \boldsymbol{\Psi} \equiv\left(\begin{array}{l}
\phi \\
\psi
\end{array}\right) \\
& B_{T} \equiv b_{T}-\left(\phi^{\prime}+\frac{2 q \sqrt{1-\epsilon^{2}}}{1+\beta} \psi^{\prime}\right) \epsilon \lambda_{T}, \quad B_{S} \equiv b_{S}-\left(\frac{\psi^{\prime}}{\beta}+\frac{2 q \sqrt{1-\epsilon^{2}}}{1+\beta} \phi^{\prime}\right) \epsilon \lambda_{T}, \\
& b_{T} \equiv \frac{1}{\lambda_{T}}+\lambda_{T}+\frac{2 \sqrt{\beta} \alpha q \sqrt{1-\epsilon^{2}} \lambda_{S}}{1+\beta}, \quad b_{S} \equiv-\frac{\sqrt{\beta} \alpha}{\lambda_{S}}+\frac{\alpha \lambda_{S}}{\sqrt{\beta}}+\frac{2 q \sqrt{1-\epsilon^{2}} \lambda_{T}}{1+\beta}, \\
& \boldsymbol{P} \equiv\left(\begin{array}{cc}
1-2 \epsilon^{2} & \frac{2 \beta q \sqrt{1-\epsilon^{2}}}{1+\beta} \\
\frac{2 q \sqrt{1-\epsilon^{2}}}{1+\beta} & 1
\end{array}\right), \quad \boldsymbol{R} \equiv\left(\begin{array}{cc}
1-\epsilon^{2} & q \sqrt{1-\epsilon^{2}} \\
q \sqrt{1-\epsilon^{2}} & 1
\end{array}\right)
\end{aligned}
$$

and a summation is implied on the repeated index $i=1,2,3$.

The first variation of $\mathscr{L}[\boldsymbol{u}, \boldsymbol{\Theta}]$ demands that the optimal fields, denoted by the subscript asterisk, satisfy the Euler-Lagrange equations,

$$
\begin{array}{r}
\nabla \cdot \boldsymbol{u}_{*}=0, \quad 2 \nabla^{2} \boldsymbol{u}_{*}+R_{T}^{1 / 2}\left(B_{T} \theta_{*}+B_{S} \sigma_{*}\right) \hat{z}-\nabla \Pi=0, \\
\boldsymbol{P} \boldsymbol{\Psi}^{\prime \prime}+R_{T}^{1 / 2} w_{*}\left(\begin{array}{c}
B_{T} \\
B_{S}
\end{array}\right)+2 \boldsymbol{R} \nabla^{2} \boldsymbol{\Theta}_{*}=0 .
\end{array}
$$

For the stationary fields to be maximizers, the second variation of $\mathscr{L}[\boldsymbol{u}, \boldsymbol{\Theta}]$ requires

$$
\left\langle|\nabla \hat{\boldsymbol{u}}|^{2}\right\rangle+\left\langle\frac{\partial \hat{\boldsymbol{\Theta}}^{T}}{\partial x_{i}} \boldsymbol{R} \frac{\partial \hat{\boldsymbol{\Theta}}}{\partial x_{i}}\right\rangle-R_{T}^{1 / 2}\left\langle\left(B_{T} \hat{\theta}+B_{S} \hat{\sigma}\right) \hat{w}\right\rangle \geqslant 0
$$

where the hat denotes deviations from the stationary fields. If we now set

$$
f \equiv \hat{\theta} \sqrt{1-\epsilon^{2}}+q \hat{\sigma}
$$

then (4.18) can be expanded into

$$
\left\langle|\nabla \hat{\boldsymbol{u}}|^{2}\right\rangle+\left\langle|\nabla f|^{2}\right\rangle+\left(1-q^{2}\right)\left\langle|\nabla \hat{\sigma}|^{2}\right\rangle-R_{T}^{1 / 2}\left\langle\frac{B_{T}}{\sqrt{1-\epsilon^{2}}} f \hat{w}+\left(B_{S}-\frac{q B_{T}}{\sqrt{1-\epsilon^{2}}}\right) \hat{\sigma} \hat{w}\right\rangle \geqslant 0 .
$$

In order to ensure that the third term is not negative, we must choose $|q| \leqslant 1$. 
The most general version of our variational problem is now to find the smallest possible value of the extremal Nusselt number, $\mathscr{L}\left[\boldsymbol{u}_{*}, \boldsymbol{\Theta}_{*}\right]$, subject to the EulerLagrange equations (4.16)-(4.17) and condition (4.19). At our disposal in this optimization are the various Lagrange multipliers and the choices of the background fields. Plasting \& Kerswell (2003) have used a general formulation of this kind in bounding the thermal convection problem. Here, we proceed less ambitiously and consider a less optimal, but certainly more straightforward version of the problem.

\subsection{Reduction to a more familiar formulation}

The general variational formulation can be reduced to a more familiar form if we make two further assumptions. First, following Doering \& Constantin (1996), we simplify the solution of the Euler-Lagrange equations by taking $\boldsymbol{u}_{*}=\mathbf{0}$. Therefore, $\boldsymbol{\Theta}_{*}=\boldsymbol{\Theta}_{*}(z)$, with

$$
\boldsymbol{\Theta}_{*}^{\prime}=-\frac{1}{2} \boldsymbol{R}^{-1} \boldsymbol{P} \boldsymbol{\Psi}^{\prime}
$$

Second, by analogy with energy stability theory, we impose the constraints

$$
q b_{T}=\sqrt{1-\epsilon^{2}} b_{S} \text { and } q B_{T}=\sqrt{1-\epsilon^{2}} B_{S},
$$

which have the advantage of eliminating the final term in (4.19), leaving

$$
\left\langle|\nabla \hat{\boldsymbol{u}}|^{2}\right\rangle+\left\langle|\nabla f|^{2}\right\rangle+\left(1-q^{2}\right)\left\langle|\nabla \hat{\sigma}|^{2}\right\rangle-B_{T} \sqrt{\frac{R_{T}}{1-\epsilon^{2}}}\langle f \hat{w}\rangle \geqslant 0 .
$$

The second relation in (4.21) also connects the two background fields to one another:

$$
\psi^{\prime}=\frac{\left(\beta+2 \epsilon^{2}-1\right) \beta q \phi^{\prime}}{\left(\beta+1-2 q^{2} \beta\right) \sqrt{1-\epsilon^{2}}} .
$$

The extremal value of the heat flux, $N u_{*}$, can now be written in the form

$$
N u_{*}=\mathscr{L}\left[\mathbf{0}, \boldsymbol{\Theta}_{*}\right]=1+\left\langle\boldsymbol{\Psi}^{\prime} T \boldsymbol{M} \boldsymbol{\Psi}^{\prime}\right\rangle,
$$

where

$$
\boldsymbol{M} \equiv\left(\begin{array}{cc}
\epsilon^{2} & 0 \\
0 & 0
\end{array}\right)+\frac{1}{4} \boldsymbol{P}^{T}\left(\boldsymbol{R}^{-1}\right)^{T} \boldsymbol{P},
$$

and the positive-definiteness of $\boldsymbol{R}^{-1}$ makes the bound, $N u_{*}$, bigger than or equal to unity. Note that (4.23) implies that $\left\langle\boldsymbol{\Psi}^{\prime T} \boldsymbol{M} \boldsymbol{\Psi}^{\prime}\right\rangle$ can be written formally in terms of a parameter-dependent coefficient times $\left\langle\phi^{\prime 2}\right\rangle$.

At this stage, the variational problem amounts to locating the smallest value of $N u_{*}$ such that (4.22) holds. If we insist that $|q|<1$, then we may simply omit the term $\left\langle|\nabla \sigma|^{2}\right\rangle$ leaving a formulation much like that explored for the Rayleigh-Bénard problem (with, once again, $f$ playing the role of temperature). The problem posed, however, is more complicated because of the richer structure of the coefficients in both the second-variation constraint (4.22) and the maximum Nusselt number (4.24).

Although any background field for which the second-variation condition is satisfied will furnish a valid upper bound, some profiles may lead to a better bound than others. Hence, it is desirable to find that background which not only satisfies the second variation but also leads to the lowest bound. Such an exercise involves a nonlinear functional optimization problem. In the next subsection, we reduce this optimization problem to an algebraic one by using piecewise linear background profiles. Before making this selection, however, we remark briefly on the choices in (4.21). These selections have the advantage of reducing the general variational 


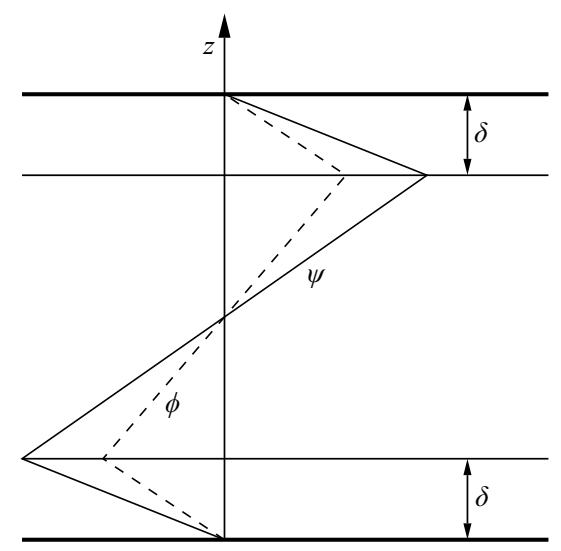

FIGURE 2. $T$ and $S$ background profiles.

formulation to something closer to the familiar Rayleigh-Bénard problem. Better still, because they also coincide with the choices made in energy stability theory, the bound is guaranteed to reduce to the energy stability condition when $R_{T}<R_{T c}$. Moreover, one can show that these selections are, in fact, the best possible choices if the background fields are piecewise linear, as in our main computations. Nevertheless, for general backgrounds and above the energy stability threshold, we cannot judge the optimality of the selection, which exposes a flaw in the current theory; one possible consequence is mentioned later.

\subsection{Piecewise linear background fields}

We now reformulate the variational problem in purely algebraic terms by introducing the piecewise linear background fields,

$$
\boldsymbol{\Psi}(z)= \begin{cases}-\left(\frac{1}{2 \delta}-1\right) \boldsymbol{\Psi}_{\text {in }}^{\prime} z, & 0 \leqslant z \leqslant \delta \\ \boldsymbol{\Psi}_{\text {in }}^{\prime}\left(z-\frac{1}{2}\right), & \delta \leqslant z \leqslant 1-\delta \\ -\left(\frac{1}{2 \delta}-1\right) \boldsymbol{\Psi}_{\text {in }}^{\prime}(z-1), & 1-\delta \leqslant z \leqslant 1,\end{cases}
$$

where $\delta(0 \leqslant \delta \leqslant 1 / 2)$ is loosely referred to as the 'boundary-layer thickness', and $\boldsymbol{\Psi}_{\text {in }}^{\prime}$ denotes the slopes of the two backgrounds in the interior region $(\delta<z<1-\delta)$. Because of (4.23), the components of the latter are not independent of one another. The shapes of the brackground fields are illustrated in figure 2.

The next step is to make the sign-indefinite term in (4.22) as small as possible. We achieve this by choosing $\boldsymbol{\Psi}_{\text {in }}^{\prime}$ so that $B_{T}=B_{S}=0$ in the interior, which demands that

$$
\boldsymbol{\Psi}_{\text {in }}^{\prime}=\frac{1}{\epsilon \lambda_{T}} \boldsymbol{S}^{-1}\left(\begin{array}{l}
b_{T} \\
b_{S}
\end{array}\right),
$$

where

$$
\mathbf{S} \equiv\left(\begin{array}{lll}
1 & 2 q \frac{\sqrt{1-\epsilon^{2}}}{1+\beta} \\
2 q \frac{\sqrt{1-\epsilon^{2}}}{1+\beta} & \frac{1}{\beta}
\end{array}\right)
$$


We are then left with only boundary layer contributions to the sign-indefinite term, but these hopefully remain controlled and small because $\hat{\boldsymbol{\Theta}}$ and $\hat{\boldsymbol{u}}$ vanish on the boundaries.

The inequality in (4.22) can now be written as

$$
\left\langle|\nabla \hat{\boldsymbol{u}}|^{2}\right\rangle+\left\langle|\nabla f|^{2}\right\rangle+\left(1-q^{2}\right)\left\langle|\nabla \hat{\sigma}|^{2}\right\rangle-\frac{b_{T}}{2 \delta} \sqrt{\frac{R_{T}}{1-\epsilon^{2}}}\langle f \hat{w}\rangle_{\mathrm{bl}} \geqslant 0,
$$

where

$$
\langle\cdots\rangle_{\mathrm{bl}} \equiv \lim _{t \rightarrow \infty} \frac{1}{4 L_{x} L_{y} t} \int_{0}^{t} \int_{-L_{y}}^{L_{y}} \int_{-L_{x}}^{L_{x}}\left(\int_{0}^{\delta} \cdots \mathrm{d} z+\int_{1-\delta}^{1} \cdots \mathrm{d} z\right) \mathrm{d} x \mathrm{~d} y \mathrm{~d} t .
$$

For convenience, we replace (4.29) by the constraint

$$
\left\langle|\nabla \hat{\boldsymbol{u}}|^{2}\right\rangle_{\mathrm{bl}}+\left\langle|\nabla f|^{2}\right\rangle_{\mathrm{bl}}-\frac{b_{T}}{2 \delta} \sqrt{\frac{R_{T}}{1-\epsilon^{2}}}\langle f \hat{w}\rangle_{\mathrm{bl}} \geqslant 0 .
$$

which is sufficient for (4.29) to be satisfied, and depends on the integrals of $\hat{\boldsymbol{u}}$ and $f$ only over the boundary layers. Hence the interior region can be omitted completely from the analysis, noting only that $\hat{\boldsymbol{u}}$ and $f$ should be smooth there. The inequality can be cast as the variational problem

$$
\frac{2 \delta}{\left|b_{T}\right|} \sqrt{\frac{1-\epsilon^{2}}{R_{T}}} \leqslant \max _{f, \hat{\boldsymbol{u}}}\langle f \hat{w}\rangle_{\mathrm{bl}} \quad \text { for } \quad\left\langle|\nabla \hat{\boldsymbol{u}}|^{2}\right\rangle_{\mathrm{bl}}+\left\langle|\nabla f|^{2}\right\rangle_{\mathrm{bl}}=1, \quad \nabla \cdot \hat{\boldsymbol{u}}=0,
$$

with $f$ and $\hat{\boldsymbol{u}}$ vanishing at $z=0$ and $z=1$ and free at $z=\delta$ and $z=1-\delta$. The EulerLagrange equations corresponding to this maximization are identical to the linear stability equations obtained for thermal convection with a layer of height $2 \delta$ and an equilibrium temperature gradient of unity. Thus, the results from thermal convection can be adapted using a suitable rescaling of the variables. Doing that, we obtain the following constraint on $\delta$ :

$$
\delta<\delta_{\max }=\frac{\sqrt{1-\epsilon^{2}}}{\left|b_{T}\right|} \sqrt{\frac{R_{c}}{R_{T}}} .
$$

Finally, we simplify the bound on the Nusselt number:

$$
N u_{*}=1+\left(\frac{1}{2 \delta}-1\right) \boldsymbol{\Psi}_{\mathrm{in}}^{\prime T} \boldsymbol{M} \boldsymbol{\Psi}_{\mathrm{in}}^{\prime} .
$$

Since we would like to obtain the smallest $N u_{*}$, we choose the biggest $\delta$ allowed by (4.33), and arrive at

$$
N u_{*}=1+\frac{b_{T}^{2}\left[1-\beta q^{2}(2-\beta)\right]}{4 \epsilon^{2} \lambda_{T}^{2}\left(1-\epsilon^{2}\right)\left(1-q^{2}\right)}\left(\frac{1}{2 \delta}-1\right),
$$

where

$$
\delta= \begin{cases}\delta_{\max }, & \delta_{\max }<\frac{1}{2} \\ \frac{1}{2}, & \delta_{\max } \geqslant \frac{1}{2} .\end{cases}
$$

This leaves us with a choice of the constants $\lambda_{T}, \lambda_{S}, \epsilon$ and $q$, which are constrained by (4.21) and must be selected to minimize $N u_{*}$ :

$$
N u_{\max }=1+\min _{\lambda_{T}, \lambda_{s}, \epsilon, q} \frac{b_{T}^{2}\left[1-\beta q^{2}(2-\beta)\right]}{4 \epsilon^{2} \lambda_{T}^{2}\left(1-\epsilon^{2}\right)\left(1-q^{2}\right)}\left(\frac{1}{2 \delta}-1\right),
$$


(a)
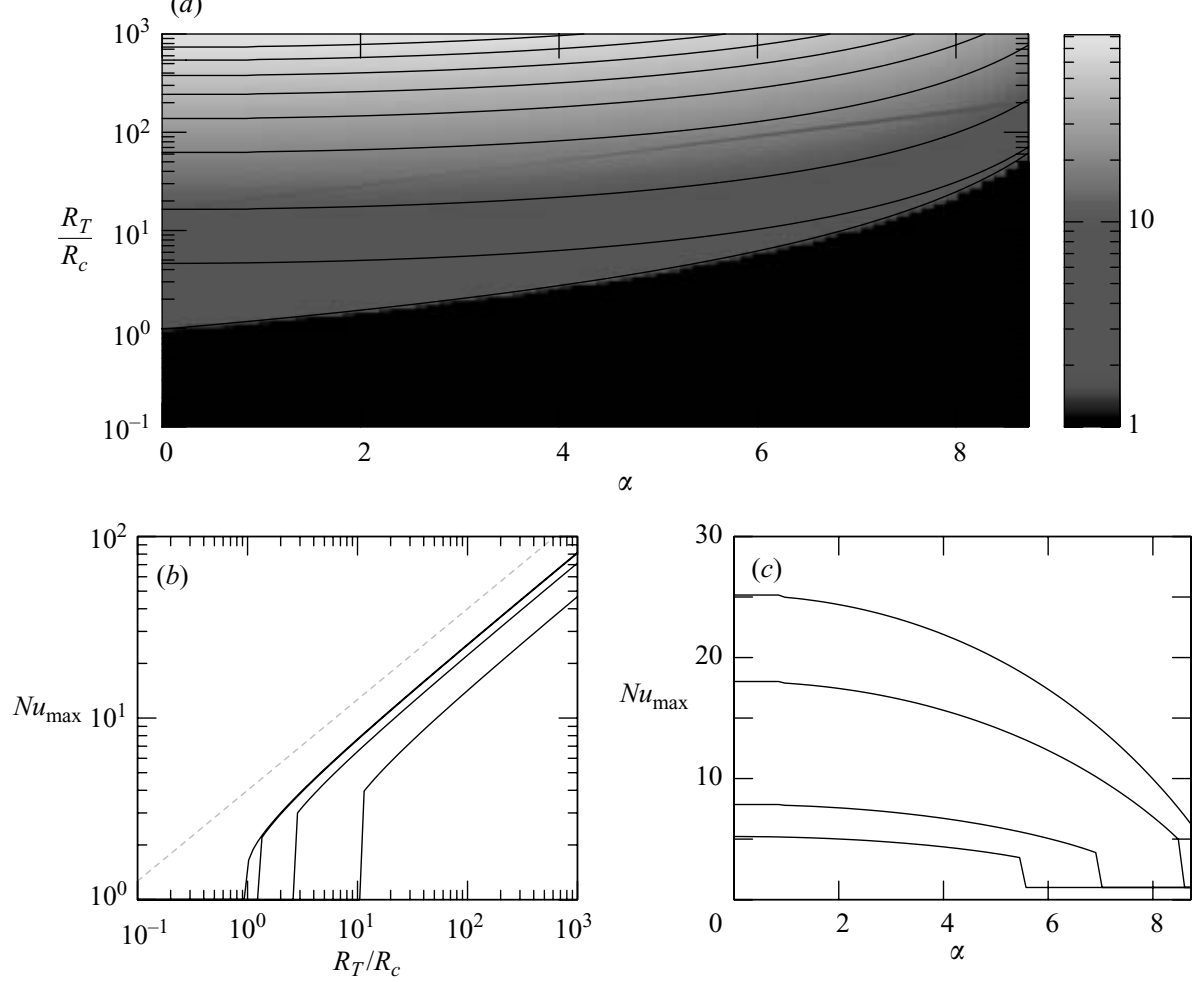

FiguRE 3. (a) The bound on Nusselt number for ODD convection, shown as a density on the $\left(\alpha, R_{T} / R_{c}\right)$-plane for $\beta=0.1$. The solid lines are contours of constant $N u_{\max }$ for values of 70 (topmost), 60, 50, 40, 30, 20, 10 and 5 (last but one), and the lowermost solid line corresponds to the energy stability threshold $R_{T}=R_{T c}$. (b) The bound for $\alpha=0$ (topmost solid), 1, 4, 7 (lowermost) as a function of $R_{T} / R_{c}$. The dotted line shows a $R_{T}^{1 / 2}$ scaling for comparison. In (c) the effect of $\alpha$ is shown for $R_{T} / R_{c}=5$ (lowermost), 10, 50 and 100 (uppermost).

subject to $q b_{T}=b_{S} \sqrt{1-\epsilon^{2}},-1<q<1$ and $0<\epsilon<1$. If $\delta_{\max } \geqslant 1 / 2$ for a suitable choice of the parameters, we set $\delta=1 / 2$ and, consequently, $N u_{\max }=1$. The condition for that to happen coincides with energy stability.

\section{Results}

The optimization in (4.37) to find the lowest upper bound on the Nusselt number is performed numerically. We made extensive use of the Matlab function fminsearch for this purpose. The results for the ODD convection and the fingering case are presented separately.

\subsection{ODD convection}

Figure 3 shows the typical behaviour of the bound for ODD convection using $\beta=0.1$. Figure $3(b)$ demonstrates that the scaling of the bound is $R_{T}^{1 / 2}$ for fixed $\alpha$, as $R_{T}$ becomes large, which can be extracted from (4.37) simply by observing the limiting dependence, $\delta \sim R_{T}^{-1 / 2}$, in the constraint (4.33). The $1 / 2$ scaling mirrors the equivalent result in the Rayleigh-Bénard problem, and one might at first sight guess that little has been gained. In fact, much more information is included in the $\alpha$-dependent prefactor to the scaling, which does not yield to asymptotic analysis and must be computed numerically. For example, an increase of $\alpha\left(R_{S}\right)$ at fixed $R_{T}$ lowers the bound, as can 


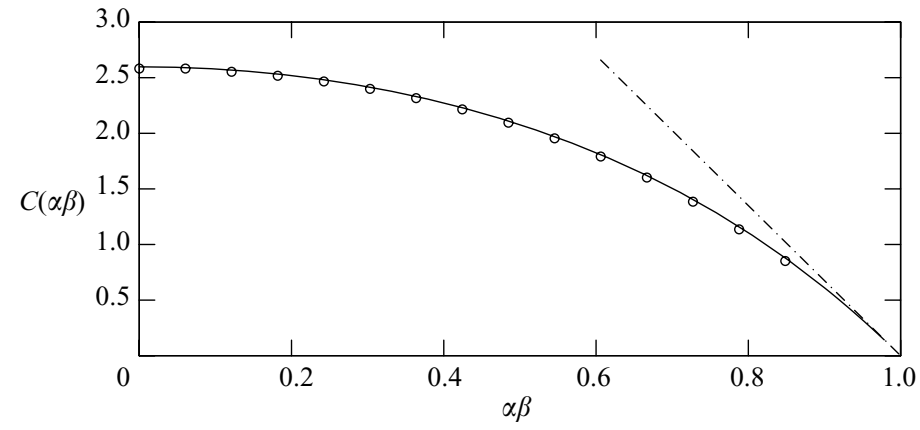

FIGURE 4. The coefficient of $\left(R_{T} / R_{c}\right)^{1 / 2}$ in the bound for $\beta \ll 1$. The solid curve is the result of the analysis given in the text. The circles correspond to the data shown in figure 3 for $R_{T}=1000 R_{c}$. The dashed line shows the asymptotic result for $\alpha \beta \sim 1, C(\alpha \beta) \sim 27(1-\alpha \beta) / 4$.

be seen in figure 3(c). The bound continues to decrease smoothly as $\alpha$ is increased, until this parameter reaches the threshold for energy stability, whereupon the bound discontinuously jumps to unity. Thus, the $\alpha$-dependence of the bound encapsulates the ability of the stabilizing component to turn off convection completely.

Although the optimization must in general be performed numerically, there is one particular limit in which we can make further progress: $\beta \ll 1$ (which is relevant to the oceanic application, where $\beta \approx 10^{-2}$ ). We begin by writing the bound as

$$
\begin{aligned}
N u_{*}-1 & =\frac{b_{T}^{2}\left[1-\beta q^{2}(2-\beta)\right]}{4 \epsilon^{2} \lambda_{T}^{2}\left(1-\epsilon^{2}\right)\left(1-q^{2}\right)}\left(\frac{1}{2 \delta}-1\right) \\
& \leqslant \frac{b_{T}^{2}\left[1-\beta q^{2}(2-\beta)\right]}{4 \epsilon^{2} \lambda_{T}^{2}\left(1-\epsilon^{2}\right)\left(1-q^{2}\right)} \frac{1}{2 \delta}=\frac{b_{T}^{3}\left[1-\beta q^{2}(2-\beta)\right]}{8 \epsilon^{2} \lambda_{T}^{2}\left(1-\epsilon^{2}\right)^{3 / 2}\left(1-q^{2}\right)} \sqrt{\frac{R_{T}}{R_{c}}},
\end{aligned}
$$

and find the values of $\lambda_{T}, q$ and $\epsilon$ that minimize the coefficient of $\sqrt{R_{T} / R_{c}}$. Guided by energy stability theory, we set $\alpha \beta \sim O(1)$. In this limit, the constraint (4.21) gives $\lambda_{S}=-\sqrt{\beta}$ and

$$
b_{T}=\frac{1}{\lambda_{T}}+\lambda_{T}-2 \alpha \beta \chi
$$

where $\chi=q \sqrt{1-\epsilon^{2}}$. We minimize (5.1) with respect to $\lambda_{T}$ to obtain

$$
\lambda_{T}=-2 \alpha \beta \chi+\sqrt{4 \alpha^{2} \beta^{2} \chi^{2}+5},
$$

which then leads to

$$
N u_{*}-1 \leqslant \frac{3^{3}}{5^{5}} \frac{\left(-3 \alpha \beta \chi+\sqrt{4 \alpha^{2} \beta^{2} \chi^{2}+5}\right)^{3}\left(2 \alpha \beta \chi+\sqrt{4 \alpha^{2} \beta^{2} \chi^{2}+5}\right)^{2}}{\epsilon^{2}\left(1-\epsilon^{2}\right)^{1 / 2}\left(1-\chi^{2}-\epsilon^{2}\right)} \sqrt{\frac{R_{T}}{R_{c}}} .
$$

This expression is optimized for

$$
\epsilon^{2}=\frac{7}{10}-\frac{3 \chi^{2}}{10}+\left[\frac{9}{100}\left(1+\chi^{2}\right)^{2}-\frac{\chi^{2}}{5}\right]^{1 / 2}
$$

which leaves $N u_{*}$ as a function of only $\chi$. The final minimization in $\chi$ must be done numerically. The result is $N u_{\max }=C(\alpha \beta) \sqrt{R_{T} / R_{c}}$, where the function $C(\alpha \beta)$ is plotted in figure 4. At $\alpha \beta=0$, the coefficient takes the value for thermal convection, $C(0)=\sqrt{27 / 4}$, and then decreases smoothly to zero as $\alpha \beta$ approaches 1 (the energy stability condition for $\left.R_{T} / R_{c} \rightarrow \infty\right)$. Also included in the figure are the results of the 

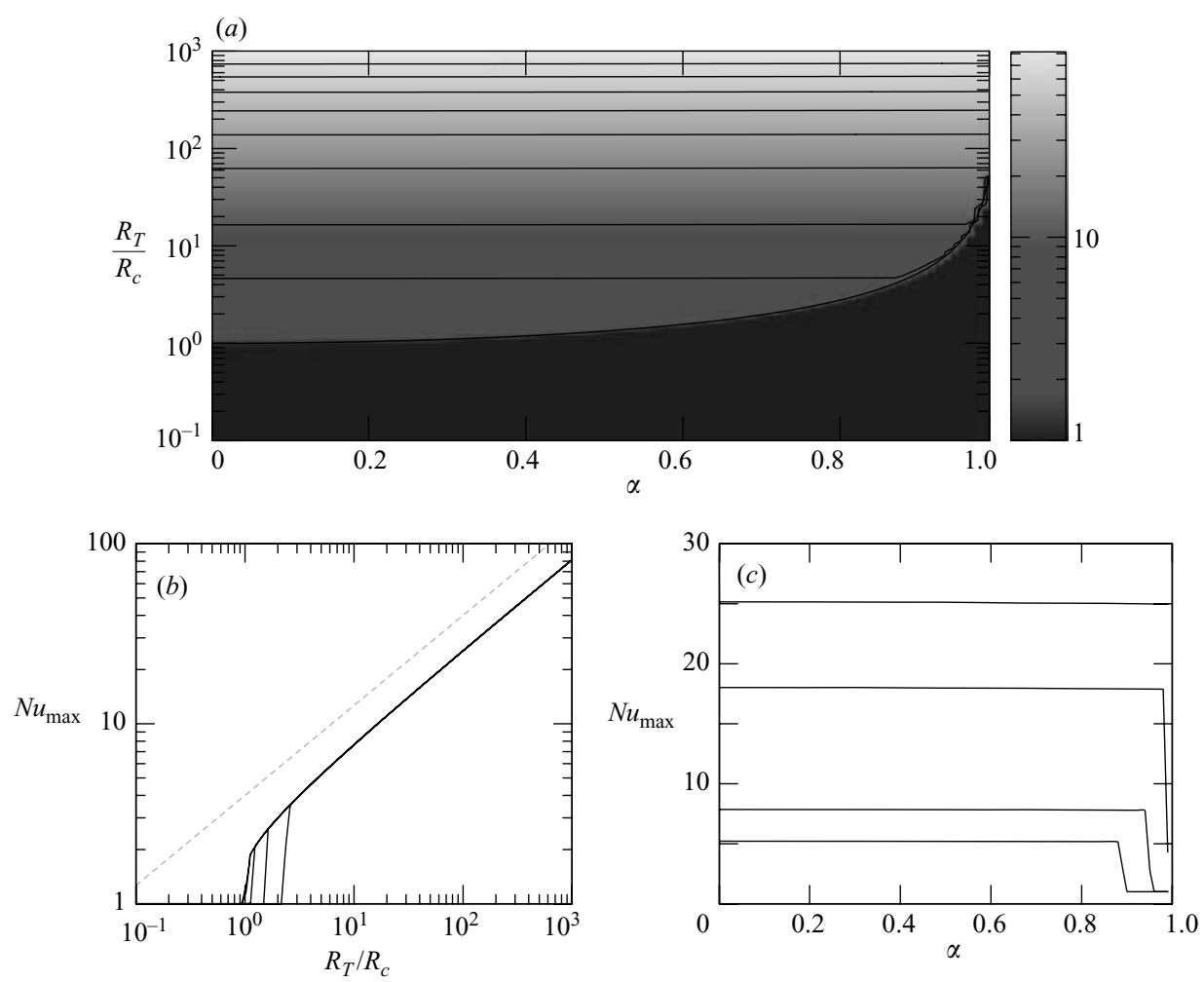

Figure 5. (a) The bound computed for $\beta=10$ (T-fingers). The solid lines are contours of constant $N u_{\max }$ for values of 70 (topmost), 60, 50, 40, 30, 20, 10, 5 (lowest but one) and the lowest solid line shows the energy stability threshold $R_{T}=R_{T c}$. $(b)$ The bound for $\alpha=0$ (topmost solid), 1, 4, 7 (lowermost) as a function of $R_{T} / R_{c}$. The dotted line shows $R_{T}^{1 / 2}$ for scaling. In (c), the effect of $\alpha$ is shown for $R_{T} / R_{c}=100$ (topmost), 50, 10 and 5 (lowermost).

full numerical optimization for $\beta=0.1$ and $R_{T}=1000 R_{c}$, which display quantitative agreement with the limiting solution.

\section{2. $T$-fingers}

The bound for $\beta=10$ is plotted in figure 5. As is clear from this picture, the asymptotic behaviour of the bound is again $R_{T}^{1 / 2}$ for large $R_{T}$, and, once more, $N u_{\max }$ is discontininuous at the energy stability boundary. A closer look reveals a relatively weak dependence of the bound on $\alpha$. Indeed, the bound obtained for $\alpha=0$ is a very good approximation to the bound for other values of $\alpha$. Figure 6 shows the dependence of the bound on $\alpha$ and $\beta$ for fixed $R_{T}=1000 R_{c}$, and illustrates again how $N u_{*}$ is only weakly sensitive to $\alpha$ in the limit of large $\beta$. Thus, we infer that, with the constraints employed and the family of backgrounds chosen, the bound is not reduced on adding the stabilizing component in this limit. Perhaps Straus' asymptotic solution of the $S$-equation could be used to improve the situation.

\subsection{Discontinuity in the bound}

There are two obvious reasons why the bounds computed above could be discontinuous on the energy stability curve, neither of which is correct. First, a discontinuity can arise due to the appearance of new finite-amplitude solutions in a saddle-node bifurcation. Indeed, the loss of energy stability at the point where the saddle-node first 


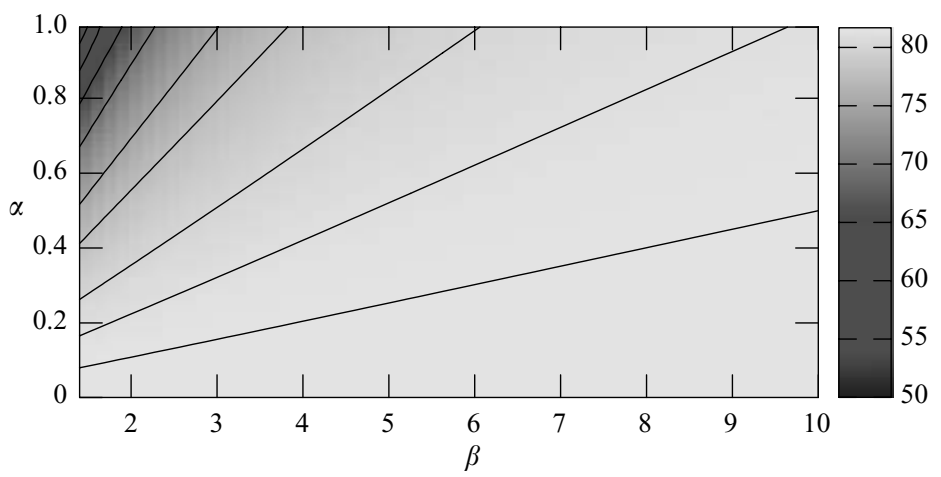

FIGURE 6. The bound $\left(N u_{\max }\right)$ computed for the range $1.4<\beta<10$ for $R_{T}=1000 R_{c}$. The solid lines show contours of constant $N u_{\max }$ for values of 81.5 (lowermost), 81, 80, 77.5, 75, 70, 65, 60 and 55 (topmost).

appears (see figure 1) suggests that a jump of this kind might well be present around these parameter settings. In this way, the bounding machinery could prove an effective tool for exploring the nonlinear dynamics of the system. Unfortunately, it turns out that the bound jumps discontinuously even in cases where there is no saddle-node and the energy stability condition coincides with linear onset (as for the fingering case). Moreover, no qualitative change occurs in the extent of the discontinuity when we approach parameter settings for which we know a saddle-node exists. Thus, the discontinuity observed in our computations does not appear to be caused primarily by the appearance of new nonlinear solutions.

The second reason why the bound could be discontinuous is that the background profiles change from being linear to piecewise linear on passing through the energy stability curve. In fact, for this reason, discontinuities exist in bounds for thermal convection. As shown by Doering \& Constantin (1996), those discontinuities can be removed by using a smoother background profile near the energy stability threshold, which raises the question of whether we can smooth out the current discontinuity by similar means.

To address this question one can return to the formulation of the variational problem in $\S 4.2$. Near the energy stability threshold, it is possible to develop asymptotic solutions via perturbation theory without choosing a particular background. The final value of the bound depends on integrals of various functions that are related to the background fields, and one could, in principle, optimize the procedure to find the best bound. However, it becomes immediately clear on heading down this avenue that the bound always jumps discontinuous at the energy stability boundary, irrespective of the choice of background. The reason can be traced to the conditions in (4.21) which, in combination with the solution of the Euler-Lagrange equations in (4.20), lead to the optimal Nusselt number in (4.24). The trouble is that the matrix $\boldsymbol{R}$ becomes singular at the energy threshold (where $q \rightarrow 1$ and $\epsilon \rightarrow 0$ ), and with the choice (4.21) already made, there is no way to adjust the background fields to ensure that $\boldsymbol{\Psi}_{*}$ remains regular there. The result is that $N u_{*}-1$ always converges to a non-zero value as $R_{T}$ approaches $R_{T c}$ from above. Given the failure of the perturbation expansion, it seems clear that the only possible way in which the discontinuity might be eliminated is by avoiding one of the two extra assumptions made at the beginning of $\S 4.2$ (namely $\boldsymbol{u}_{*}=\mathbf{0}$ or (4.21)). 


\section{Discussion and open questions}

In this work, we have bounded fluxes in double-diffusive convection using the Constantin-Doering-Hopf background method. Of particular interest is the behaviour of the bound for large Rayleigh numbers, where we find the dependence $R_{T}^{1 / 2}$. This bound is different from empirical flux laws often quoted in the literature (Turner 1965), which show $N u \sim R_{T}^{1 / 3}$. One reason for this discrepancy is that our bound may simply be too conservative and grossly overestimate the physically realized flux, as can be seen in comparison with the some numerical and asymptotic solutions (Radko \& Stern 2000). Indeed, many examples of double-diffusive convection in the laboratory and ocean show the formation of internal boundary layers (salt finger interfaces, diffusive steps), yet our optimal backgrounds only exhibit such sharp features next to the walls and do not capture whatever process is responsible. However, as is also true in Rayleigh-Bénard problem, it is not clear whether the observed flows have converged to the ultimate asymptotic state of double-diffusive convection. If that state is characterized by flux laws which do not depend explicitly on the molecular values of diffusivity and viscosity, a $1 / 2$ scaling law must eventually emerge.

A main difficulty addressed in this article is to account for the effect of the stabilizing element on the bound. This effect disappears from the most straightforward implementation of the background method, as it does from regular energy stability theory. A similar problem is posed for geophysical and astrophysical systems in a rotating frame of reference, where there is no effect of rotation rate in standard energy stability theory and its extensions. The Prandtl number also plays no role in the bounding theory of thermal as well as double-diffusive convection. The fact that the theory does not depend on these parameters does not mean that the system is insensitive to them, but is merely a result of discarding the governing PDEs and keeping only certain integral equations derived from them. Thus, the problem facing us is to add more integral constraints in order to incorporate the missing physics (see Ierley \& Worthing 2001).

Here, we have identified and exploited a key constraint for doubly diffusive convection. The role of this constraint in energy stability theory is instructive, and amounts to generalizing the definition of the energy function so that one can suppress transient amplification in the absence of finite-amplitude instability. The constraint, however, is far from sufficient in describing all the features of double-diffusive convection. In fact, the generalized energy stability threshold still seems to fall short of where we expect nonlinear solutions to come into existence. This leaves one suspicious that there may still be inconsequential transient amplification above threshold, and prompts the two key questions: Is it possible to differentiate between such transient growth and a true finite-amplitude instability? Is it possible to improve energy analysis further so that the loss of energy stability always signifies a linear or nonlinear instability?

The bound we have derived is discontinuous along the energy stability boundary. Such jumps could reflect the appearance of additional finite-amplitude solutions at saddle-node bifurcations, an eventuality that certainly occurs for double-diffusive convection. Unfortunately, our numerical computations offer little evidence that this is the main cause of the discontinuity. The jump could also have been introduced because we have used piecewise linear background fields. Forcing the backgrounds to be smooth removes any discontinuity of this kind in the Rayleigh-Bénard problem. For the current problem, however, the difficulty is far more insidious: one can establish for the simplified variational formulation in $\S 4.2$ that the bound remains discontinuous even for smooth background fields. The only remaining possibility for 
further progress in using the bounding machinery to detect saddle-node bifurcations is to retain the more general version variational problem in $\S 4.1$.

Finally, the background method is geared towards extending energy stability theory to find the properties of the solution with the biggest norm. While this method has provided us with some useful insight, other modifications of energy stability theory must also be possible. In particular, it is conceivable that one may be able to incorporate thresholds on the norm of perturbations that decay to the trivial state, thus allowing one to extend the energy stability threshold for sufficiently 'small' disturbances. Such a method could address important issues like the abrupt transition to turbulence in some shear flows. Double-diffusive convection remains a rich testing ground for all such future developments.

We acknowledge NSF grant ATM0222109 and a discovery NSERC grant for support. We thank Charles Doering and Richard Kerswell for discussions and suggestions. The authors were hosted by the Department of Mathematics, MIT, and the GFD summer program, WHOI, at various times during this work and the hospitality is gratefully acknowledged.

\section{Appendix. Energy stability}

Starting with (3.20), we consider two cases.

$$
\text { Case 1: } \beta<1
$$

We substitute

$$
\lambda_{T}=k_{T} \sqrt{\frac{1+\beta}{1-\beta}} \quad \text { and } \quad \lambda_{S}=k_{S} \sqrt{\frac{\beta(1+\beta)}{1-\beta}}
$$

into the constraint (3.17), to obtain

$$
k_{T}-\frac{1}{k_{T}}=-\alpha\left(k_{S}-\frac{1}{k_{S}}\right) .
$$

By letting $A \equiv k_{S}-1 / k_{S}$, (A 2) leads to the following relations:

$$
k_{T}=\frac{-\alpha A \pm \sqrt{\alpha^{2} A^{2}+4}}{2} \text { and } k_{S}=\frac{A \pm \sqrt{A^{2}+4}}{2} .
$$

We seek the largest $R_{T}$ for nonlinear stability which satisfies (3.19). Therefore, we would like to minimize $\left|b_{T}\right|$. By substituting (A 1) and (A 3) in (3.12), we see that the best choice to make $\left|b_{T}\right|$ as small as possible is when the signs of the second terms of $k_{T}$ and $k_{S}$ in (A 3$)$ are different. Therefore,

$$
\left|b_{T}\right|=\frac{1}{\sqrt{1-\beta^{2}}}\left|\sqrt{\alpha^{2} A^{2}+4}-\beta \alpha \sqrt{A^{2}+4}\right| .
$$

Case 1a: $\alpha \leqslant \beta$

$b_{T}$ attains the minimum when

$$
A^{2}=\frac{4}{\alpha^{2}} \frac{\beta^{2}-\alpha^{2}}{1-\beta^{2}}
$$

which gives $\left|b_{T}\right|=2 \sqrt{1-\alpha^{2}}$. From (3.19) and the definition of $\alpha$, we obtain

$$
R_{T}-R_{S}<R_{c} \text {. }
$$


Case $1 b: \beta \leqslant \alpha \leqslant 1 / \beta$

$b_{T}$ attains the minimum when $A=0$, which gives $b_{T}=2(1-\beta \alpha) / \sqrt{1-\beta^{2}}$. In this case, we obtain

Case $1 c: \alpha \geqslant 1 / \beta$

$$
\sqrt{R_{T}}-\beta \sqrt{R_{S}}<\sqrt{1-\beta^{2}} \sqrt{R_{c}}
$$

In this case $\left|b_{T}\right|=0$ because we may choose

$$
A^{2}=\frac{4\left(\beta^{2} \alpha^{2}-1\right)}{1-\beta^{2}} \text {. }
$$

Therefore, the system is nonlinear stable for all values of $R_{T}$.

$$
\text { Case 2: } \beta>1
$$

Here, we substitute

$$
\lambda_{T}=k_{T} \sqrt{\frac{\beta+1}{\beta-1}} \quad \text { and } \quad \lambda_{S}=k_{S} \sqrt{\frac{\beta(\beta+1)}{\beta-1}}
$$

into the constraint (3.17) to obtain

$$
k_{T}+\frac{1}{k_{T}}=-\alpha\left(k_{S}+\frac{1}{k_{S}}\right) .
$$

By letting $A \equiv k_{S}+1 / k_{S}$, (A 9) leads to the following relations:

$$
k_{T}=\frac{-\alpha A \pm \sqrt{\alpha^{2} A^{2}-4}}{2} \text { and } k_{S}=\frac{A \pm \sqrt{A^{2}-4}}{2} .
$$

By substituting (A 8) and (A 10) in (3.12) and choosing different signs of the second terms of $k_{T}$ and $k_{S}$ in (A 10), we obtain

$$
\left|b_{T}\right|=\frac{1}{\sqrt{\beta^{2}-1}}\left|\beta \alpha \sqrt{A^{2}-4}-\sqrt{\alpha^{2} A^{2}-4}\right| .
$$

Case 2a: $\alpha<1$

$\left|b_{T}\right|$ attains the minimum when

$$
A^{2}=\frac{4}{\alpha^{2}} \frac{\beta^{2}-\alpha^{2}}{\beta^{2}-1}
$$

which gives $\left|b_{T}\right|=2 \sqrt{1-\alpha^{2}}$. We then obtain

$$
R_{T}\left(1-\alpha^{2}\right)<R_{c}
$$

or

$$
R_{T}-R_{S}<R_{c}
$$

Case $2 b: \alpha \geqslant 1$

By substituting

$$
A^{2}=\frac{4}{\alpha^{2}} \frac{\beta^{2} \alpha^{2}-1}{\beta^{2}-1},
$$

in (A 11), we obtain $\left|b_{T}\right|=0$. It is straightforward to show that $\alpha \geqslant 1$ is a sufficient and necessary condition for $A^{2} \geqslant 4$. Therefore, the system is nonlinearly stable for all values of $R_{T}$. 


\section{REFERENCES}

Baggett, J. S., Driscoll, T. A. \& Trefethen, L. N. 1993 A mostly linear model of transition to turbulence. Phys. Fluids 7, 833-838.

Baines, P. G. \& Gill, A. E. 1969 On thermohaline convection with linear gradients. J. Fluid Mech. 37, 289-306.

BRYAN, F. 1986 High latitude salinity effects and interhemispheric thermohaline circulations. Nature 323, 301-304.

Busse, F. H. 1969 On Howard's upper bound for heat transport by turbulent convection. J. Fluid Mech. 37, 457-477.

Dijkstra, H. A., Te RaA, L. \& Weijer, W. 2004 A systematic approach to determine thresholds of the ocean's thermohaline circulation. Tellus 56, 362-370.

Doering, C. R. \& Constantin, P. 1996 Variational bounds on energy dissipation in incompressible flows. III. Convection. Phys. Rev. E 53, 5957-5981.

Howard, L. N. 1963 Heat transport by turbulent convection. J. Fluid Mech. 17, 405-432.

IERLEY, G. R. \& Worthing, R. A. 2001 Bound to improve: A variational approach to convective heat transport. J. Fluid Mech. 441, 223-253.

Jacobs, C. A., Huppert, H. E., Holdsworth, G. \& Drewry, D. J. 1981 Thermohaline steps induced by melting at the Erebus Glacier toungue. J. Geophys. Res. 86, 6547-6555.

JosePH, D. D. 1976 Stability of Fluid Motions, vol. II. Springer.

Kerswell, R. R. 1998 Unification of variational principles for turbulent shear flows: The background method of Doering-Constantin and the mean-fluctuation formulation of HowardBusse. Physica D 121, 175-192.

LiNDBERG, W. R. 1971 An upper bound on transport processes in turbulent thermohaline convection. J. Phys. Oceanogr. 1, 187-195.

Malkus, W. V. R. 1954 The heat transport and specturm of thermal turbulence. Proc. R. Soc. Lond. A $225,196-212$.

Merryfield, W. J. 2000 Origin of thermohaline staircases. J. Phys. Oceanogr. 30, 1046-1068.

Neal, V. T., Neshyba, S. \& Denner, W. 1969 Thermal stratification in the Arctic Ocean. Science 166, 373-374.

Nicodemus, R., Grossmann, S. \& Holthaus, M. 1997 Improved variational principle for bounds on energy dissipation in turbulent shear flow. Physica D 101, 178-196.

Plasting, S. C. \& Kerswell, R. R. 2003 Improved upper bound on the energy dissipation rate in plane Couette flow: the full solution to Busse's problem and the Constantin-Doering-Hopf problem with one-dimensional background field. J. Fluid Mech. 477, 363-379.

RAdKo, T. \& Stern, M. E. 2000 Finite-amplitude salt fingers in a vertically bounded layer. J. Fluid Mech. 425, 133-160.

RuDDICK, B. 1992 Intrusive mixing in a Mediterranean salt lens - intrusion slopes and dynamical mechanisms. J. Phys. Oceanogr. 22, 1274-1285.

Schmitt, R. W. 1994 Double diffusion in oceanography. Annu. Rev. Fluid Mech. 26, 255-285.

Spiegel, E. A. 1969 Semiconvection. Comm. Astrophys. Space Phys. 1, 57-60.

STERn, M. E. 1969 Collective instability of salt fingers. J. Fluid Mech. 35, 209-218.

StERN, M. E. 1982 Inequalities and variational-principles in double-diffusive turbulence. J. Fluid Mech. 114, 105-121.

Stocker, T. F. 1999 Abrupt climate changes: from the past to the future - a review. Intl J. Earth Sci. 88, 365-374.

Straus, J. M. 1973 Upper bound on the solute flux in double diffusive convection. Phys. Fluids 17, $520-527$.

Turner, J. S. 1965 Buoyancy Effects in Fluids. Cambridge University Press.

Tziperman, E. \& Ionnnou, P. J. 2002 Transient growth and optimal excitation of thermohaline variability. J. Phys. Oceanogr. 32, 3427-3435.

UlRICH, R. K. 1972 Thermohaline convection in stellar interiors. Astrophys. J. 172, 165-177.

VAuClaIR, S. 2004 Thermohaline convection and metallic fingers in polluted stars. In The A-Star Puzzle (ed. J. Zverko, J. Ziznovsky, S. Adelman \& W. Weiss), Proc. IAU Symposium 224, p. $161-166$.

Veronis, G. 1965 On finite amplitude instability in thermohaline convection. J. Mar. Res. 23, 1-17.

WALEFFE, F. 1995 Transition in shear flows - Nonlinear normality versus nonnormal linearity. Phys. Fluids 7, 3060-3066. 